\title{
Operation-Switchable Bidirectional Pulsed Fiber Laser Incorporating Carbon-Nanotube-based Saturable Absorber
}

\author{
H. H. Liu, Student Member, IEEE, and K. K. Chow, Senior Member, IEEE
}

\begin{abstract}
We present an operation-switchable bidirectional ring-cavity pulsed fiber laser incorporating a carbon-nanotube -based saturable absorber and a polarization-dependent four-port circulator. By manipulating the intra-cavity polarization state of light, two sets of mode-locked pulses in terms of fundamental repetition rate and pulse width can be achieved separately from the same laser cavity. In clockwise direction, the laser generates mode-locked output pulse train with a pulse width of $600 \mathrm{fs}$ and a fundamental repetition rate of $12.68 \mathrm{MHz}$. While in counter-clockwise direction, the laser generates mode-locked pulses with a pulse width of $480 \mathrm{fs}$ and a fundamental repetition rate of 16.46 $\mathrm{MHz}$. Furthermore, the same laser cavity can produce bidirectional Q-switched output pulse trains with synchronized repetition rate. Such synchronized repetition rate is linearly proportional to pump power. The results show a multi-functional pulsed fiber laser with selectable output pulse trains.
\end{abstract}

Index Terms - optical fiber lasers, optical materials, optical pulse generation

\section{INTRODUCTION}

$\mathrm{F}$ iber lasers generating optical pulses with short duration and high energy have emerged as one of best light sources in a broad range of applications including frequency metrology, microscopy, micromachining, ranging finding, and sensing [1, 2]. In general, two mechanisms are involved for fiber lasers to generate pulses: mode-locking and Q-switching. Mode-locked fiber lasers generally produce soliton-like pulses in anomalous net cavity dispersion through the interplay between fiber dispersion and nonlinearity [3]. Such pulses have typical duration down to sub-picoseconds [4]. On the other hand, Q-switched fiber lasers produce pulses only when the population inversion of gain medium at its highest level is larger than the cavity loss [2]. Q-switched fiber lasers are capable of generating pulses with pulse energy much higher than that generated from mode-locked fiber lasers [5]. As the

Manuscript received XXX. This work was partially supported by Academic Research Fund Tier 1 Grant (RG 22/10) of Ministry of Education and Nanyang Technological University, Singapore.

H. H. Liu and K. K. Chow are with School of Electrical and Electronic Engineering, Nanyang Technological University, 50 Nanyang Avenue, Singapore 639798 (e-mail: kkchow@ntu.edu.sg). two types of lasers can produce pulses with extinguished features, the design of one multi-functional fiber laser to generate versatile types of pulse trains is much attractive. By inserting an isolator into the laser cavity, the pulsed lasers can operate in unidirectional cavity configuration. In 2009, K. H. Lin et al. demonstrated the generation of Q-switched pulse train as well as mode-locked pulse train from a unidirectional fiber laser incorporating hybrid saturable absorbers (SAs) [6]. Distinct from the unidirectional cavity configuration, the bidirectional cavity configuration enables pulsed lasers to operate without isolators. Such bidirectional mode-locked lasers emitting ultrashort pulses in the opposite directions are very useful for gyroscopic sensors applications [7, 8]. Also, bidirectional Q-switched lasers emitting pulse trains with difference wavelength and synchronized repetition rate in the opposite directions have potential applications in various fields including sum-frequency mixing [9] and synthesis of optical waveform [10, 11]. In 2011, Ouyang et al. demonstrated that the bidirectional fiber laser incorporating two semiconductor saturable absorber mirrors (SESAMs) can simultaneously emit two soliton-like pulse trains with distinct fundamental repetition rates in the opposite directions [12]. Recently, Y. Cui et al. demonstrated that the bidirectional fiber laser mode-locked by graphene and carbon nanotubes (CNTs) based SAs can separately generate dissipative and conventional solitons [13].

In recent years, CNT-based SAs have been extensively explored in pulsed fiber laser due to its advantages including fast recovery time, wide operation bandwidth with tube diameter control, compatible to fiber, and capacity to work in bidirectional modes $[14,15]$. In this work, we demonstrate an operation-switchable bidirectional pulsed fiber laser incorporating a CNT-based SA and a polarization-dependent four-port circulator. By controlling the intra-cavity polarization state of light, two counter-circulating mode-locked pulse trains with different features are enabled from the same laser cavity. In the clockwise (CW) direction, the laser generates mode-locked output pulse train with a pulse width of $600 \mathrm{fs}$ and a fundamental repetition rate of $12.68 \mathrm{MHz}$. While in the counter-clockwise (CCW) direction, the laser generates mode-locked pulse train with a pulse width of $480 \mathrm{fs}$ and a fundamental repetition rate of $16.46 \mathrm{MHz}$. Furthermore, the 
laser can operate in Q-switching state in both CW and CCW directions. An output pulse train with pulse energy of $18.7 \mathrm{~nJ}$ in the CW direction and that with pulse energy of $23.3 \mathrm{~nJ}$ in the CCW direction are found to be synchronized at the same repetition rate of $8 \mathrm{kHz}$. Such synchronized repetition rate is found to be linearly proportional to pump power.

\section{BIDIRECTIONAL PULSED FIBER LASER INCORPORATING CNT-BASED SA}

Fig. 1(a) shows the experimental setup of the all-fiber bidirectional laser incorporating a CNT-based SA. A 0.63-m-long piece of erbium-doped fiber (EDF: LIEKKI Er80) with a group velocity dispersion (GVD) parameter $\beta_{2}$ of $-0.022 \mathrm{ps}^{2} / \mathrm{m}$ is pumped by a $976-\mathrm{nm}$ laser diode via a $980 / 1550$ $\mathrm{nm}$ wavelength-division multiplexing (WDM) coupler. The WDM coupler comes with 0.62-m-long HI1060 fiber pigtails with $\beta_{2}$ of $+0.02 \mathrm{ps}^{2} / \mathrm{m}$. A fiber based polarization controller

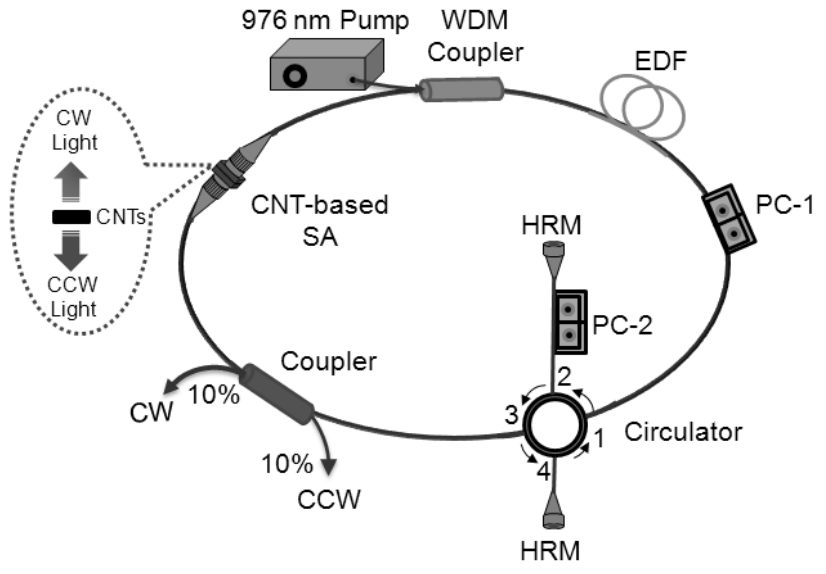

(a)

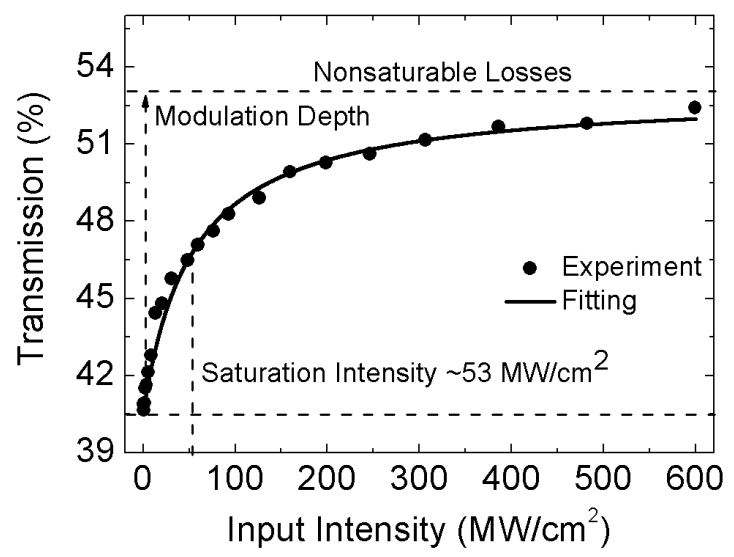

(b)

Fig. 1. (a) Schematic diagram of all-fiber bidirectional laser incorporating carbon-nanotube- (CNT) based saturable absorber (SA) and (b) transmission characteristics of the CNT-based SA. WDM: wavelength-division multiplexing; EDF: erbium-doped fiber; PC: polarization controller; HRM: highly reflected mirror; CW: clockwise; CCW: counter-clockwise.
(PC-1) after the EDF is used to adjust and optimize the polarization condition of the common laser cavity. A polarization-dependent four-port circulator with polarization maintaining (PM) fiber pigtails is adopted. In the cavity, light passes through the circulator following the configuration $1 \rightarrow 2,2 \rightarrow 3,3 \rightarrow 4$, and $4 \rightarrow 1$. Ports 1 and 3 are directly spliced to the common laser cavity, where port 2 is spliced to a fiber based polarization controller (PC-2) and then connected to a highly reflected mirror, which forms the arm- 1 for $\mathrm{CW}$ directional operation. Port 4 with 1.77-m-long polarization maintaining fiber pigtail is connected to another highly reflected mirror, which serves as arm-2 for CCW directional operation. A $10 / 90$ fiber coupler is used to extract $10 \%$ of power from the common laser cavity as the laser output for each direction. The total lengths of the cavity in CW direction and CCW direction are around $16.2 \mathrm{~m}$ and $12.5 \mathrm{~m}$, respectively. Note that the net cavity GVD in each direction is anomalous which enables soliton shaping in the laser.

The CNT-based SA is constructed by optically-driven deposition of CNTs to form a thin film on a FC/APC connector [16]. The SA is finished by connecting another FC/APC connector by a conventional adaptor. In our experiment, the CNTs are synthesized by high-pressure CO conversion (HiPCO) and dispersed in dimethylformanide (DMF) solvent. The CNTs show a desirable absorption peak near C-band [17]. The nonlinear absorption of the prepared CNT-based SA is first characterized by a conventional pulsed fiber laser with a center wavelength of $1562 \mathrm{~nm}$. Such laser delivers pulse trains with pulse width of $260 \mathrm{fs}$, repetition rate of $68.27 \mathrm{MHz}$ and maximum average power of $20 \mathrm{~mW}$. Fig. 1(b) shows the measurement result of the nonlinear transmission of the prepared CNT-based SA. The modulation depth and nonsaturable losses are calculated to be $12 \%$ and $47.5 \%$, respectively. The saturation intensity is estimated to be $\sim 53$ $\mathrm{MW} / \mathrm{cm}^{2}$.

\section{BIDIRECTIONAL MODE-LOCKING OPERATION}

The laser operation can be optimized by adjusting PC-1 and PC-2 as there is polarizer effect in the four-port circulator. By controlling the polarization state of PC-1, the intra-cavity power in the common laser can be optimized where the total output power in both $\mathrm{CW}$ direction and CCW direction are maximized. While changing the polarization state of PC-2, the operation state of laser can be tuned. In the experiment, we always fix the PC-1 after the optimization of the intra-cavity power and only adjust the polarization state of PC-2. The pump threshold for mode-locking in the CW direction is found to be $\sim 26.7 \mathrm{~mW}$ together with adjusting polarization state of PC-2. The output power is measured around $126.5 \mu \mathrm{W}$ in the CW direction while it is only $\sim 2 \mu \mathrm{W}$ in CCW direction under the pump power of $26.7 \mathrm{~mW}$. Fig. 2(a) shows the spectral profile of the laser output from the CW direction. The center wavelength of optical spectrum is $1562.58 \mathrm{~nm}$ with a 3-dB bandwidth of 4.3 $\mathrm{nm}$. The sharp sidebands periodically superimposed on the pulse spectrum clearly indicate that soliton-like pulses are 
generated in the laser cavity [3]. Fig. 2(a) also depicts the autocorrelation trace of the output pulse with a resolution of 25 fs. The pulse width is estimated to be $600 \mathrm{fs}$ with a sech ${ }^{2}$ fitting and the time-bandwidth product is calculated to be 0.317 , which is nearly transform-limited. The RF spectrum of the output pulse is also given in Fig. 2(b). The output pulse train exhibits an extinction ratio of $80 \mathrm{~dB}$ at the fundamental repetition rate of $12.68 \mathrm{MHz}$. Moreover, no sidebands within a wide frequency range are observed as shown in the inset of Fig. 2(b), which further confirms that stable mode-locking is obtained. When the pump power is further increased to $32 \mathrm{~mW}$ or above, multiple pulses are observed due to the soliton energy quantization [18]. In the operation state of CW directional mode-locking, the characterization of optical properties of the CCW directional laser is impossible due to its extremely low output power.

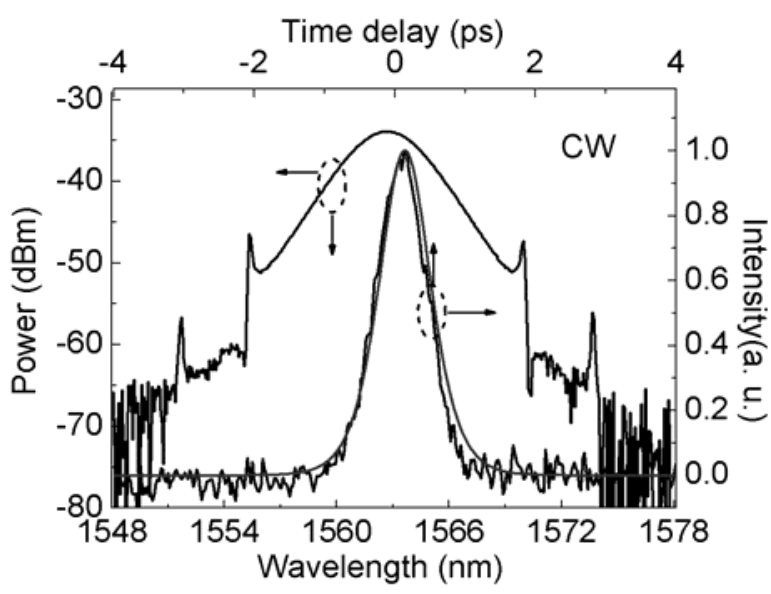

(a)

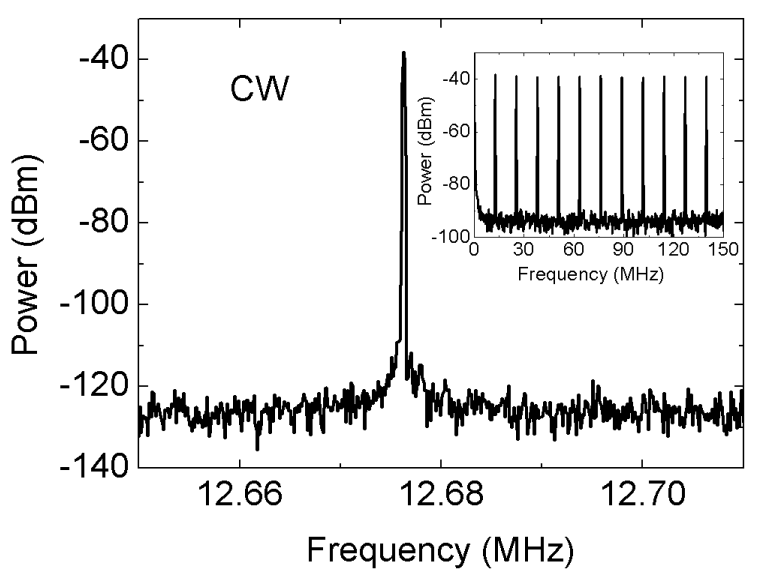

(b)

Fig. 2. (a) Output optical spectrum and autocorrelation trace of the CNT-based bidirectional pulsed laser in CW direction, and (b) the corresponding RF spectrum with the inset showing the RF spectrum in a wider frequency range.

By further changing the polarization state of PC-2 and tuning the pump power to $30.9 \mathrm{~mW}$, the single-soliton generation in the CCW direction can be obtained. The corresponding output power is measured to be $246 \mu \mathrm{W}$ from the CCW direction with that of $0.4 \mu \mathrm{W}$ from the $\mathrm{CW}$ direction. Fig. 3(a) shows the output optical spectrum from the CCW direction showing a center wavelength of $1562.08 \mathrm{~nm}$ and a 3-dB bandwidth of 5.4 $\mathrm{nm}$. Kelly-sidebands are superimposed on the soliton spectrum. The pulse width is estimated to be 480 fs with a sech ${ }^{2}$ fitting and the time-bandwidth product is calculated to be 0.319 . Fig. 3(b) gives the corresponding RF spectrum of the laser output. An extinction ratio of $90 \mathrm{~dB}$ at the frequency of $16.46 \mathrm{MHz}$ shows that the laser operates in fundamental cavity frequency. The RF spectrum in a wider frequency range is also given in the inset of Fig. 3(b). Once the laser is mode-locked, it shows considerable stable operations for hours. By further increasing pump power to $34 \mathrm{~mW}$, multiple pulses are produced from the laser cavity.

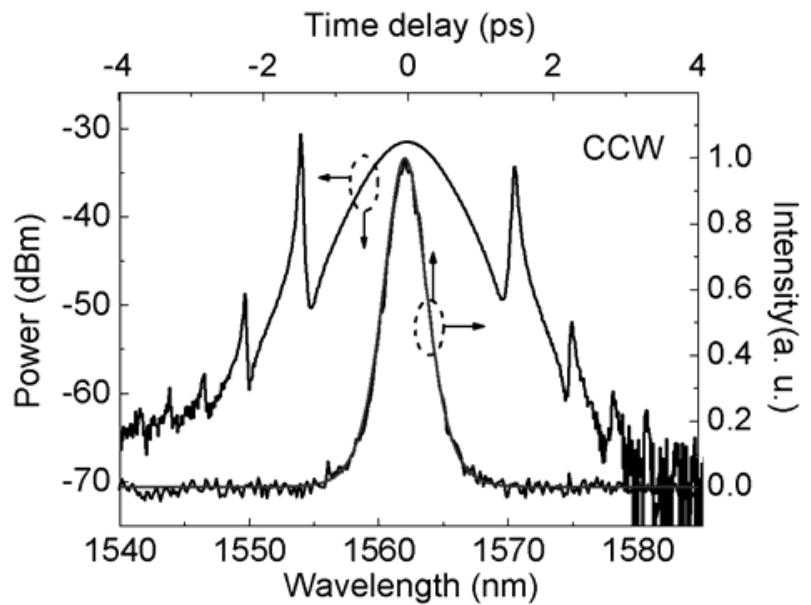

(a)

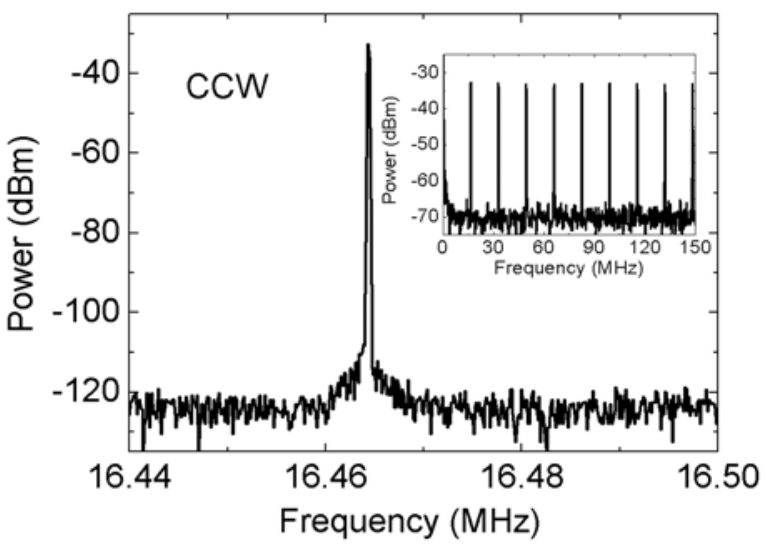

(b)

Fig. 3. (a) Output optical spectrum and autocorrelation trace of the CNT-based bidirectional pulsed laser in CCW direction, and (b) the corresponding RF spectrum with the inset showing the RF spectrum in a wider frequency range.

\section{BIDIRECTIONAL Q-SWITCHING OPERATION}

When the output power from both CW and CCW directions are adjusted to have similar levels, the laser switches to operate 
in bidirectional Q-switching state. Fig. 4(a) shows the output pulse trains of the laser with a pump power of $30.3 \mathrm{~mW}$. It is found that the pulse trains from the two directions are synchronized and exhibit a common repetition rate of $\sim 8 \mathrm{kHz}$. There is little amplitude modulation shown on the pulse envelopes in Fig. 4(a), illustrating that the effect of self-mode-locking on the Q-switching operation is suppressed [19]. The duration of the Q-switched pulses in both directions is estimated to be $10 \mu \mathrm{s}$. The corresponding output powers are measured to be $149.6 \mu \mathrm{W}$ and $186.6 \mu \mathrm{W}$ in $\mathrm{CW}$ direction and CCW direction, respectively. Thus, the pulse energies are calculated to be $18.7 \mathrm{~nJ}$ and $23.3 \mathrm{~nJ}$, respectively. It is worth mentioning that the obtained pulse energy is higher than current reports on the passively Q-switched fiber lasers which incorporate CNT-based thin-film SAs [19, 20]. For conventional ring-cavity fiber lasers that operate in Q-switching and prohibit from broadband mode-locking, the cavity configurations need SAs as well as narrowband filters such as fiber Bragg grating which might sacrifice pulse energy. In our experiment, a relatively high energy bidirectional Q-switched ring-cavity fiber laser is achieved with a

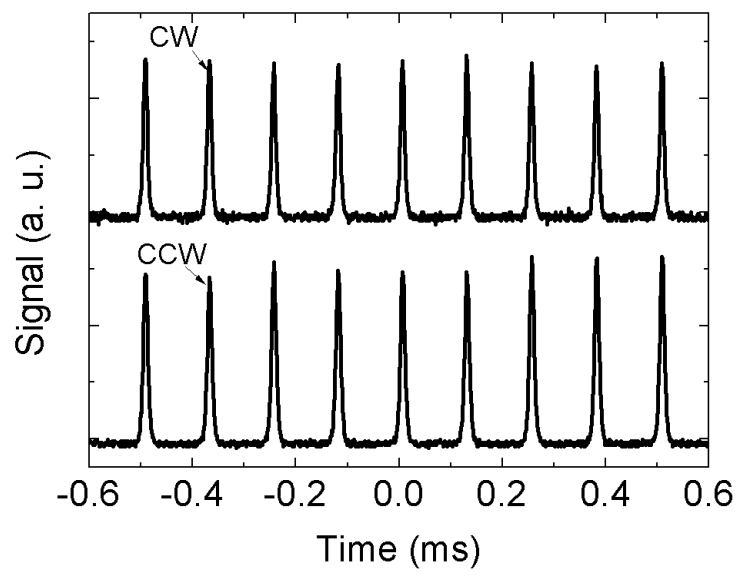

(a)

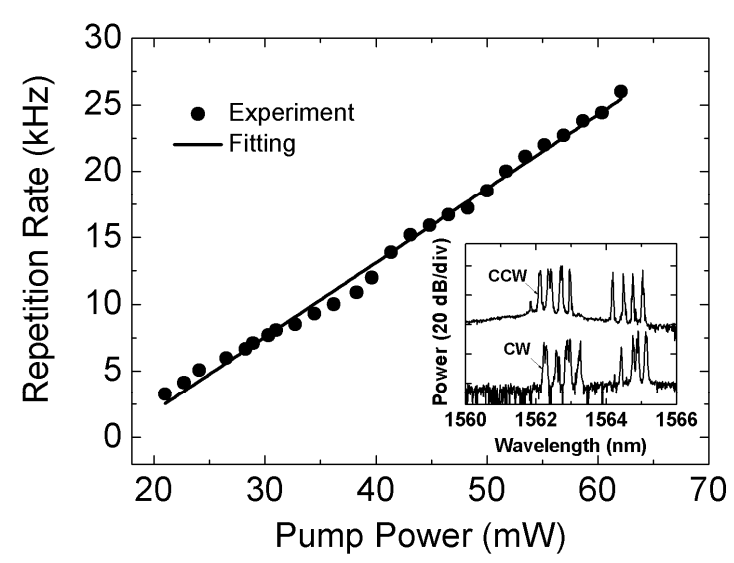

(b)

Fig. 4. (a) Output of CW direction (upper) and CCW direction (lower) of the bidirectional Q-switched pulse trains with synchronized repetition rate, and (b) plot of the synchronized repetition rate against pump power; the inset shows the offset optical spectra of Q-switched pulse trains in CW direction and CCW direction.
CNT-based SA with no lasing mode selector. The optical spectra of Q-switched pulse trains in CW direction and CCW direction under pump power of $30.3 \mathrm{~mW}$ are measured simultaneously as in the inset of Fig. 4(b). Different from the unidirectional mode-locked laser with large spectral bandwidth, multi-wavelength emission with narrow spectral bandwidth is observed for bi-directional Q-switched lasers. It is found that the emitted wavelengths in CW direction are mostly suppressed in CCW direction and vice versa, which reveals that the gain competition (or mode competition) could take place in the opposite directions. The synchronized repetition rate is linearly proportional to pump power as shown in Fig. 4(b). When the pump power is $\sim 60 \mathrm{~mW}$, the repetition rate is raised to $\sim 25.8$ $\mathrm{KHz}$.

\section{DISCUSSION}

The investigation on the tunable operation states of the developed bidirectional fiber laser is further performed by adjusting polarization state of PC-2. Fig. 5 plots the power-splitting ratio of the CCW direction against the total output power of both directions. It is found that the power-splitting ratio keeps nearly constant as the total power increases by increasing the pump power. When the power-splitting ratio is greater than $80 \%$, the laser falls in CCW directional mode-locking. When the power-splitting ratio is less than $20 \%$ the laser operates in CW directional mode-locking. Once mode-locking is achieved in one direction, light circulating in the opposite direction is strongly suppressed although there is no obvious isolator embedded in the laser cavity. Such unidirectional mode-locking with high suppression of the power-splitting ratio in the opposite direction could be explained by gain competition when the emitted light dominates in one direction. By tuning the power-splitting ratio in the range of $20 \%-80 \%$, the laser only

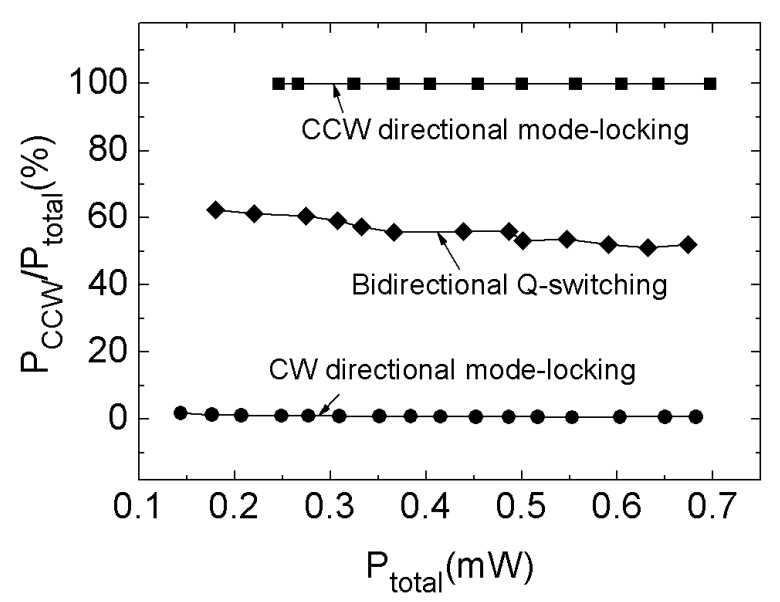

Fig. 5. Plot of power-splitting ratio against total output power of the fiber laser with different operation states. 
produces bidirectional Q-switched pulse trains with synchronized repetition rate even though under high pump power. The laser operating in Q-switching rather than mode-locking is believed to be a result of the mutual saturation of the thin-film CNT-based SA by incident light beam in the opposite directions. Since the SA has low absorption at high incident power, the loss experienced by two pulses passing through the SA simultaneously is less than that of passing through the SA separately. As a Q-switched pulse has typical pulse duration of $\sim \mu$ s which is much shorter than the cavity round-trip time of $\sim$ ns, the SA is occupied before it is hit by the pulse from the opposite direction. Such bidirectional pulses saturate SA simultaneously could result in high transmission of SAs. The laser tends to operate in state of bidirectional Q-switching due to the less intra-cavity loss. In order to achieve simultaneously mode-locking from both output ports, either a CNT-deposited tapered fiber with a long CNT-light interaction length or two active modulators have been previously reported for deferring the mutual saturation of SA in the bidirectional fiber lasers [7, 8]. In our experiment, the simultaneously mode-locking from both output ports can be obtained when CNTs are deposited on the two highly reflected mirrors for functioning as two individually reflective CNT-based SAs. When we remove the CNT-based SAs from the bidirectional fiber laser cavity, no matter how the polarization states of PCs is adjusted, only continuous wave is emitted from the two output ports within the pump power of $100 \mathrm{~mW}$. Although the effective polarization together with two PCs could act as an artificial SA via the effect of nonlinear polarization evolution, the self-started mode-locking of the laser through fiber nonlinearity is hardly achieved under the relatively low pump power $[21,22]$. Since the concrete SAs play important roles in influencing the operation regime of bidirectional pulsed lasers, future work on the investigation of the fiber laser incorporating a common CNT-based SA with various CNT-light interaction lengths is suggested.

\section{CONCLUSION}

In summary, we demonstrate an operation-switchable bidirectional ring-cavity fiber laser incorporating a carbon-nanotube-based saturable absorber and a polarization-dependent four-port circulator. By adjusting the intra-cavity polarization state, pulse trains from the opposite directions with different features are enabled from the common laser cavity. The laser can operate among states of both passive mode-locking and Q-switching. Mode-locking in the CW direction is achieved under pump power of $26.7 \mathrm{~mW}$ and the laser outputs pulses with a pulse width of $600 \mathrm{fs}$ and a repetition rate of $12.68 \mathrm{MHz}$. While the mode-locked laser in the CCW direction generates pulses with a pulse width of $480 \mathrm{fs}$ and a repetition rate of $16.46 \mathrm{MHz}$ under pump power of $30.9 \mathrm{~mW}$. Although no isolator is inserted into the laser cavity, the unidirectional mode-locking can be obtained with high suppression of the power-splitting ratio on the light circulating in the opposite direction. By carefully adjusting the power-splitting ratio in the two circulating directions, the laser can operate in the state of bidirectional Q-switching. Output pulses with energy of $18.7 \mathrm{~nJ}$ in the CW direction and that of $23.3 \mathrm{~nJ}$ in the CCW direction are found synchronized at a repetition rate of $8 \mathrm{kHz}$. Such synchronized repetition rate is linearly proportional to pump power. The results show a multi-functional pulsed fiber laser with selectable output pulse train.

\section{REFERENCES}

[1] M. E. Fermann and I. Hartl, "Ultrafast fiber laser technology,” IEEE J. Sel. Topics Quantum Electron., vol. 15, no. 1, pp. 191-206, Jan. 2009.

[2] O. Svelto, "Principles of Lasers," $4^{\text {th }}$ ed. New York: Plenum, 1998.

[3] L. E. Nelson, D. J. Jones, K. Tamura, H. A. Haus, and E. P. Ippen, "Ultrashort-pulse fiber ring lasers," Appl. Phys. B, vol. 65, no. 2, pp. 277-294, Feb. 1997.

[4] H. A. Haus, K. Tamura, L. E. Nelson, and E. P. Ippen, "Stretched-pulse additive pulse mode-locking in fiber ring lasers: theory and experiment," IEEE J. Quantum Electron., vol. 31, no. 3, pp. 591-598, Mar. 1995.

[5] J. Y. Huang, S. C. Huang, H. L. Chang, K. W. Su, Y. F. Chen, and K. F. Huang, "Passive Q switching of Er-Yb fiber laser with semiconductor saturable absorber," Opt. Express, vol. 16, no. 5, pp. 3002-3007, Feb. 2008.

[6] K. H. Lin, J. J. Kang, H. H. Wu, C. K. Lee, and G. R. Lin, "Manipulation of operation states by polarization control in an erbium-doped fiber laser with a hybrid saturable absorber," Opt. Express, vol. 17, no. 6, pp. 4806-4814, Mar. 2009.

[7] K. Kieu, and M. Mansuripur, "All-fiber bidirectional passively mode-locked ring laser,” Opt. Lett., vol. 33, no. 1, pp. 64-66, Jan. 2008.

[8] A. Braga, J. Diels, R. Jain, R. Kay, and L. Wang, "Bidirectional mode-locked fiber ring laser using self-regenerative, passively controlled, threshold gating,” Opt. Lett., vol. 35, no. 15, pp. 2648-2650, Aug. 2010.

[9] Y. F. Chen, Y. S. Chen, and S. W. Tsai, "Diode-pumped Q-switched laser with intracavity sum frequency mixing in periodically poled KTP,” Appl. Phys. B, vol. 79, no. 2, pp. 207-210, Jul. 2004.

[10] N. D. Lai, F. Bretenaker, and M. Brunel, "Coherence of pulsed microwave signals carried by two-frequency solid-state lasers,” J. Lightw. Technol., vol. 21, no. 12, pp. 3037-3042, Dec. 2003.

[11] M. Brunel and M. Vallet, "Pulse-to-pulse coherent beat note generated by a passively Q-switched two-frequency laser,” Opt. Lett., vol. 33, no. 1, pp. 2524-2526, Nov. 2008.

[12] C. Ouyang, P. Shum, K. Wu, J. H. Wong, H. Q. Lam, and S. Aditya, "Bidirectional passively mode-locked soliton fiber laser with a four-port circulator,” Opt. Lett., vol. 36, no. 11, pp. 2089-2091, Jun. 2011.

[13] Y. Cui and X. Liu, "Graphene and nanotube mode-locked fiber laser emitting dissipative and conventional solitons,” Opt. Express, vol. 21, no. 16, pp. 18969-18974, Aug. 2013.

[14] S. Y. Set, H. Yaguchi, Y. Tanaka, and M. Jablonski, "Ultrafast fiber pulsed lasers incorporating carbon nanotubes," IEEE J. Sel. Top. Quantum Electron., vol. 10, no. 1, pp. 137-146, Feb. 2004.

[15] S. Yamashita, "A tutorial on nonlinear photonic applications of carbon nanotube and graphene,” J. Lightw. Technol., vol. 30, no. 4, pp. 427-447, Feb. 2012

[16] H. H. Liu, Y. Yang and K. K. Chow, "Enhancement of thermal damage threshold of carbon-nanotube-based saturable absorber by evanescent-field interaction on fiber end," Opt. Express, vol. 21, no. 16, pp. 18975-18982, Aug. 2013.

[17] K. K. Chow, S. Yamashita, and S. Y. Set, "Four-wave-mixing-based wavelength conversion using a single-walled carbon-nanotube-deposited planar lightwave circuit waveguide,” Opt. Lett., vol. 35, no. 12, pp. 2070-2072, Jun. 2010.

[18] D. T. Yang, L. M. Zhao, B. Zhao, and A. Q. Liu, "Mechanism of multisoliton formation and soliton energy quantization in passively mode-locked fiber lasers,” Phys. Rev. A., vol. 72, no. 4, pp. 043816, Oct. 2005.

[19] D. P. Zhou, L. Wei, B. Dong, and W. K. Liu, “Tunable passively Q-switched erbium-doped fiber laser with carbon nanotubes as a saturable absorber,” IEEE Photonics Technol. Lett., vol. 22, no. 1, pp. 9-11, Jan. 2010. 
[20] B. Dong, J. Hao, J. Hu, and C. Y. Liaw, "Wide pulse-repetition-rate range tunable nanotube Q-switched low threshold erbium-doped fiber laser," IEEE Photonics Technol. Lett., vol. 22, no. 24, pp. 1853-1855, Dec. 2010

[21] D. Chernykh, A. Krylov , A. Ogleznev, V. Popok, N. Arutunan, A. Pozharov, V. Grebenyukov, E. Obraztsova, and E. Dianov, "Bidirectional ultra-short pulse erbium doped fiber ring laser with extremely wide channels' tuning", in Proc. Laser Physics Workshop (LPHYS'13), paper no. 8.3.1, Prague, 2013.

[22] K. Jiang, S. Fu, P. Shum, and C. Lin, “A wavelength-switchable Passively harmonically mode-Locked fiber laser with low pumping threshold using single-walled carbon nanotubes,” IEEE Photonics Technol. Lett., vol. 22, no. 11, pp. 754-756, Jun. 2010.

H. H. Liu (M'09) received B. Eng. and M. Eng. degrees in communication engineering from Yangzhou University and Shanghai University, China, in 2007 and 2010, respectively. She was appointed as a Research Associate in the Key Lab of Specialty Fiber Optics and Optical Access Networks at Shanghai
University in 2007. Since Jan. 2010, she pursues her doctoral degree in Nanyang Technological University (NTU), Singapore. Her current research interests focus on ultra-fast fiber lasers and carbon nano-material based optical devices.

K. K. Chow (M’02, SrM'10) received his B. Eng. (with honors), M. Phil., and $\mathrm{Ph}$. D. degrees in electronic engineering from The Chinese University of Hong Kong (CUHK), Hong Kong, in 1998, 2000, and 2003, respectively. He was a postdoctoral fellow in the same institute from 2003 to 2005. During his Ph. D. and postdoctoral work in CUHK, he specialized in fiber-based all-optical data processing devices. Since 2005, He was with Research Center for Advanced Science and Technology (RCAST), and later on Department of Electrical Engineering and Information Systems, The University of Tokyo, Japan, as a Research Fellow, specialized in advanced fiber materials and structures as well as carbon-based nano-materials for applications in photonics areas. From 2010, he has been an Assistant Professor in School of Electrical and Electronic Engineering, Nanyang Technological University, Singapore. 